\title{
OS CAMINHOS DA PESQUISA CIENTÍFICA EM ADMINISTRAÇÃO EM BUSCA DA RELEVÂNCIA PERDIDA
}

\author{
The paths of management scientific research in search of the lost relevance
}

Thomaz Wood Jr.*

Renato Jose de Souza**

\section{RESUMO}

A discussão da relevância social da pesquisa em administração tem sido tema de diversos artigos científicos. Este estudo tem como objetivo contribuir para o entendimento da evolução dessa corrente reflexiva, fornecendo um mapa com a trajetória histórica da discussão no âmbito internacional, buscando fomentar o debate no Brasil. Para isso, realizamos uma análise de rede de citação sobre uma base de 780 artigos. Identificamos os maiores citadores, os mais citados e o caminho principal, o qual revelou a trajetória do tema da relevância social da pesquisa em administração desde a década de 1960. Observamos que as distintas lógicas e perspectivas presentes na discussão resultaram em uma pluralidade de abordagens e posições. Concluímos que o campo ainda se encontra em evolução, porém os debates já indicam caminhos e soluções que podem ser incorporados às práticas de pesquisa das escolas de administração. Com base nos achados da pesquisa, nós advogamos uma orientação prática para a pesquisa brasileira: o direcionamento de esforços para a realização de pesquisas orientadas para a relevância, mantendo, entretanto, a busca do rigor científico.

Palavras-chave: Pesquisa em Administração. Relevância Social. Rigor Científico. Impacto Social. Rede de Citação. Bibliometria.

\section{ABSTRACT}

The social relevance of research in Management has been the subject of several papers. This study aims to contribute to the understanding of the evolution of this reflexive current, offering a map with a historical trajectory of this discussion in the international field and seeking to foment debates in Brazil. To achieve our goal, we performed a citation network analysis on a basis containing 780 articles. We identified the top citers, the top cites, and the main path of the network, which showed the trajectory of development for the literature in the field since the 1960s. We noted that the distinct logics present in the discussion resulted in a plurality of approaches and perspectives. We conclude that the field is still evolving. However, the debates offer paths and solutions that may be incorporated into the research practices of business schools. Based on the findings of the research, we advocate a practical orientation for Brazilian research. Such orientation might be based on efforts for the development of research aimed at relevance, maintaining, however, the pursuit of scientific rigor.

Keywords: Research in Management. Social Relevance; Scientific Rigor. Social Impact. Citation Network Analysis. Bibliometrics.

* Doutor em Administração de Empresas pela Escola de Administração de Empresas de São Paulo da Fundação Getúlio Vargas (FGV-EAESP). Professor do Programa de Pós-graduação em Administração de Empresas da Escola de Administração de Empresas de São Paulo da Fundação Getúlio Vargas (FGV-EAESP) - São Paulo (SP), Brasil. E-mail: thomaz.wood@fgv.br. ORCID: 0000-0002-5610-4699

** Doutor em Administração de Empresas pela Escola de Administração de Empresas pela Escola de Administração de Empresas de São Paulo da Fundação Getúlio Vargas (FGV-EAESP) - São Paulo (SP), Brasil. E-mail: renatojsouza@yahoo.com. ORCID: 0000-0001-7984-9047 


\section{INTRODUÇÃO}

A questão da relevância social tem sido objeto de intenso debate na Academia. Periódicos internacionais de sólida reputação dedicaram edições especiais ao tema e a algumas de suas variantes (e.g., Journal of Management Studies, 2009; Organization Studies, 2010; British Journal of Management, 2011). Em paralelo, a The Association to Advance Collegiate Schools of Business (AACSB), principal certificadora norte-americana de escolas de administração, criou um grupo tarefa para um amplo estudo sobre o impacto da pesquisa e do conhecimento produzido no âmbito das escolas de administração afiliadas à instituição (AACSB, 2008), levando à criação de diretrizes e padrões (AACSB, 2012; 2013).

Tem-se discutido o que é uma pesquisa relevante, com tentativas para atribuir características a tal tipo de pesquisa (THOMAS; TYMON, 1982; NICOLAI; SEIDL, 2010). Pode-se afirmar que uma pesquisa relevante é aquela que tem potencial de impacto e é capaz de gerar benefícios para organizações, grupos de interesse e a sociedade em geral (LIMA; WOOD, 2014). Por exemplo, uma pesquisa sobre cadeias produtivas em um setor exportador pode, eventualmente, ajudar empresas desse setor a aperfeiçoar suas práticas e melhorar o desempenho. Outro exemplo, uma pesquisa sobre o uso de tecnologia de informação na gestão pública municipal pode orientar investimentos, resultando em maximização do benefício para seus habitantes.

A discussão sobre a relevância social da pesquisa em administração tem raízes antigas. As décadas de 1940 e 1950 marcaram uma migração da discussão sobre a relevância da pesquisa nas Ciências Naturais e Tecnológicas para as Ciências Sociais e, mais especificamente, para a Administração (HAUSER, 1946). Isso ocorreu em um momento histórico no qual se discutia o princípio de que a ciência deveria atender às necessidades da população (BUSH, 1945).

Ao longo dos anos, a discussão sobre relevância social teve distintos enfoques. 0 debate central tratou da questão do rigor científico versus relevância social, acentuando o privilégio de um em relação ao outro, ou a necessidade de ambos, em diferentes momentos históricos ou correntes de pensamento (THORPE et al., 2011). Esse debate incorporou outras perspectivas como, por exemplo, aquela que enfatiza a cooperação entre universidade, setor público e indústria (ETZKOWITZ; LEYDESDORFF, 1995; 1998) ou a relação entre pesquisadores e praticantes no desenvolvimento da pesquisa socialmente relevante (RYNES; BARTUNEK; DAFT, 2001).

Ocorreu também uma discussão sobre o papel das escolas de administração na formação e no apoio às práticas gerenciais (PFEFFER; FONG, 2002; BENNIS; O'TOOLE, 2005) e uma argumentação em torno dos modos de produção do conhecimento, em especial os chamados modo 1 e modo 2. Enquanto o modo 1 prioriza o rigor científico e a publicação em periódicos com alto índice de impacto, o modo 2 se caracteriza por uma preocupação com a utilização social do conhecimento, priorizando a relevância (STARKEY, 2001; BRESNEN; BURRELL, 2012).

No Brasil, o debate associado ao tema da relevância social da pesquisa tem sido pautado por reflexões críticas sobre o sistema de medição da produção científica, sobre a qualidade desta mesma produção e sobre sua orientação produtivista (ALCADIPANI, 2011a; 
BERTERO et al., 2013). Uma busca específica, na base Scielo, considerando publicações da área de administração, de 2008 a 2017, e utilizando as palavras-chave relevância social e impacto social, resultou em apenas dois artigos (MASCARENHAS; ZAMBALDI; MORAES, 2011; LIMA; WOOD, 2014). Apesar da existência de um corpo crítico de reflexões, nós permanecemos alheios ao debate científico internacional.

Este estudo busca contribuir para o desenvolvimento de um mapa desse amplo território de investigação científica, procurando prover uma visão da evolução histórica e das correntes atuais de pesquisa no âmbito internacional. Ambiciona também fomentar 0 debate no Brasil.

O restante deste artigo está estruturado da seguinte maneira: na próxima seção, discutimos o método da análise da rede de citação; na seção seguinte, apresentamos e discutimos os resultados do estudo realizado; e na última, uma síntese do estudo, identificando as contribuições do artigo e apresentamos nossa tomada de posição sobre a pesquisa em administração no Brasil.

\section{MÉTODO}

Este estudo utilizou a análise de rede de citações (GARFIELD, 1970; 1979). Trata-se de um método bibliométrico que permite explorar relações entre autores e artigos, ligando um artigo (citado) a outro (citador). A análise da rede de citações tem sido utilizada para compreender o desenvolvimento científico de campos de pesquisa (CALERO-MEDINA; NOYONS, 2008). Em termos de procedimentos, seguimos as etapas adotadas por Georgiou (2014) e Georgiou e Heck (2015).

Para constituição da base de análise, realizamos, primeiramente, uma busca por artigos em quatro bases de dados: EBSCO, ISI Web of Knowledge, Google Scholar e Scopus. Nosso objetivo foi identificar textos sobre relevância social da pesquisa em administração. Utilizamos palavras-chave usualmente associadas ao tema, tais como: relevância, impacto social, rigor da pesquisa, pesquisa aplicada e modo 1 /modo 2 , limitando nossa busca a trabalhos publicados como artigos científicos (artigos originais ou revisões) em periódicos acadêmicos.

O principal critério de decisão para inclusão de artigos na base foi que estes deveriam tratar das questões do rigor e da relevância social da pesquisa em administração. Para determinar a inclusão ou exclusão de cada artigo, analisamos o título, resumo e palavras-chave. Quando surgia uma dúvida sobre o atendimento desse critério, analisávamos o texto no todo. Esse processo gerou 104 artigos especificamente sobre rigor e relevância da pesquisa em administração, nosso foco de interesse.

Em um segundo passo, analisamos e incorporamos à base as referências bibliográficas dos 104 artigos identificados. Este passo resultou em uma base ampliada de artigos. Então, eliminamos as redundâncias e examinamos na íntegra todos os artigos. Nessa terceira lista apenas incluímos artigos publicados em periódicos científicos. Foram, portanto, excluídos livros, capítulos de livros, anais de encontros científicos, relatórios e artigos não publicados (working papers). Esta terceira lista continha 780 artigos que haviam sido publicados entre 1876 e 2015. Tal lista constituiu a base da nossa rede de citação. 
Esta rede foi então objeto de análises utilizando o software Pajek (NOOY; MRVAR; BATAGELJ, 2011). Este software foi escolhido para a análise porque, além de ser um recurso de acesso livre e ter atualizações constantes, foi projetado para ser um calculador de rede que utiliza o método de contagem de caminhos de busca, tido como método de preferência em redes de citação (GEORGIOU, 2014; GEORGIOU; HECK, 2015). O processo compreendeu as seguintes análises: (1) visão geral da rede de citação; (2) identificação dos maiores citadores; (3) identificação dos autores mais citados; e (4) análise do caminho principal. Os resultados são apresentados e discutidos na próxima seção.

\section{RESULTADOS E DISCUSSÃO}

\section{ESTRUTURA DA REDE DE CITAÇÃO}

O Quadro 1 apresenta a distribuição dos 780 artigos identificados ao longo de oito períodos. 0 primeiro período foi definido a partir do primeiro artigo publicado, 1876, até 1949, período com menor densidade de artigos. Os seis períodos seguintes foram definidos por décadas. 0 último período foi definido a partir de 2010 até o ano do último artigo publicado, 2015. Este procedimento foi baseado em práticas adotadas em estudos anteriores semelhantes (MARTIN, 2011).

Quadro 1 - Distribuição de artigos por período

\begin{tabular}{|c|c|}
\hline Período & Número de Artigos \\
\hline $1876-1949$ & $19(2 \%)$ \\
\hline $1950-1959$ & $8(1 \%)$ \\
\hline $1960-1969$ & $36(5 \%)$ \\
\hline $1970-1979$ & $56(7 \%)$ \\
\hline $1980-1989$ & $100(13 \%)$ \\
\hline $1990-1999$ & $207(27 \%)$ \\
\hline $2000-2009$ & $278(36 \%)$ \\
\hline $2010-2015$ & $76(9 \%)$ \\
\hline Total & $780(100 \%)$ \\
\hline
\end{tabular}

A rede de citação é composta de 780 artigos (vértices) e 2717 arcos (conexões entre citadores e citados). A Figura 1 apresenta a rede de citação distribuída em oito períodos. Cada número representa um artigo. Fazendo-se uma leitura da esquerda para a direita, a figura apresenta todos os artigos incluídos na rede de citação, distribuídos em décadas (topo da figura), ligados por linhas que representam as ligações entre citadores e citados. A figura revela o aumento da densidade da rede ao longo das décadas, o que advém do aumento de publicações e do aumento do número de citações, ou seja, revela um campo em forte desenvolvimento. 


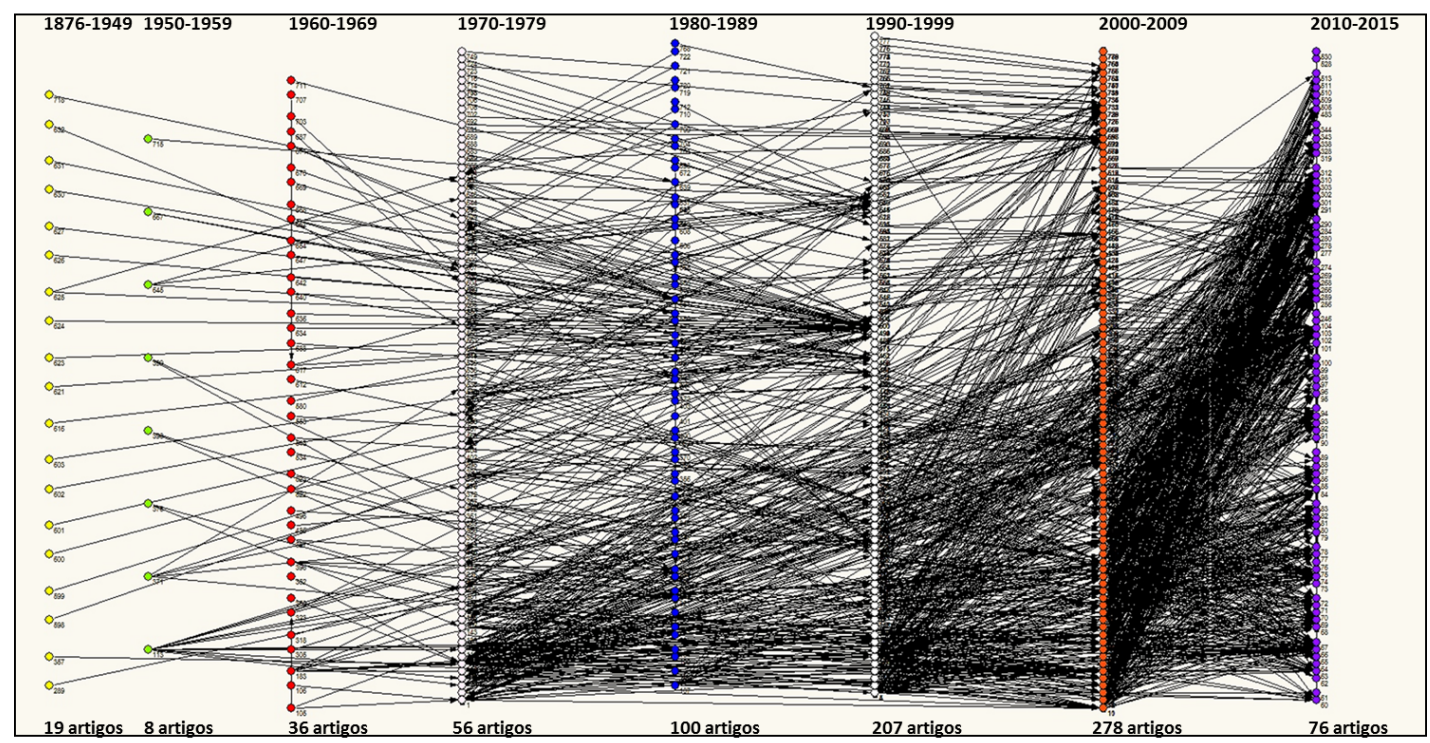

Figura 1 - Rede de citação

Notamos que a maioria dos artigos (485, representando $62 \%$ do total) foi publicada nas décadas de 1990 e 2000. Isto reflete o interesse crescente sobre a questão da relevância social da pesquisa, bem como a publicação de trabalhos seminais no início dos anos 1990 (GIBBONS et al., 1994), os quais fomentaram novos estudos.

Observou-se que, embora o número de artigos esteja em uma ascendente, os mesmos temas permeiam o debate sobre rigor e relevância da pesquisa desde o primeiro artigo publicado: "Abstract Science in America, 1776-1876" (NEWCOMB, 1876). Este artigo foi o ponto de partida na defesa da pesquisa científica com uma vocação puramente teórica, sem nenhum interesse no uso prático dos conhecimentos dela oriundos.

A análise detalhada dos artigos da rede de citação permitiu identificar três momentos históricos nos quais ocorreu o debate sobre o rigor e relevância da pesquisa. As trocas entre estes períodos foram marcadas por mudanças no conteúdo e perspectiva que norteavam o debate. 0 primeiro momento reflete questões relacionadas às Ciências Naturais e Tecnológicas, enquanto o segundo diz respeito especificamente à Administração. Por sua vez, o terceiro é marcado pela questão dos modos de produção.

\section{ANTECEDENTES (1876-1949): RELEVÂNCIA DA PESQUISA NAS CIÊNCIAS NATURAIS ETECNOLÓGICAS}

A análise da rede de citação revela, primeiramente, que o tema da relevância social da pesquisa é objeto de um debate antigo, ocorrido no âmbito das Ciências Naturais e Tecnológicas, o qual antecede aquele das Ciências Sociais, em geral, e da Administração, em particular. 
Os artigos publicados na primeira metade do século XX revelam dois aspectos importantes. Primeiro, a discussão não estava centrada na relevância da pesquisa, mas no debate sobre pesquisa pura (ou básica) versus pesquisa aplicada. A motivação do pesquisador é o que separava a ciência pura (entendimento do fenômeno) da ciência aplicada (atuação sobre o fenômeno) (CARTY, 1916; KENNELLY, 1926). Ambas eram consideradas relevantes e complementares, e uma se beneficiava da outra, embora houvesse, na época, uma maior valorização da pesquisa pura (FLINN, 1921; MILLIKAN, 1926).

Sob esta perspectiva, a ciência aplicada era o emprego da ciência pura a uma classe específica de problemas (HUXLEY, 1888). Vale lembrar que o mundo da época era marcado por grandes descobertas na física, na química e na biologia e por grandes desenvolvimentos nas engenharias. A universidade tinha um papel de liderança no desenvolvimento científico e tecnológico (MACLAURIN, 1916; BAILEY, 1926). Naquele momento, o valor da universidade era medido pelos avanços na pesquisa básica, antecedendo a pesquisa aplicada (COMPTON, 1927).

Pode-se afirmar que o debate entre pesquisa básica e pesquisa aplicada continuou e continua a permear a discussão sobre a relevância social da ciência (REAGAN, 1967; NICOLAI, 2004). Entretanto, essa discussão ganhou um caráter distinto quando a discussão chegou ao campo das Ciências Sociais, como veremos a seguir. Um segundo aspecto presente nas discussões desse período diz respeito à busca por rigor científico pelas Ciências Aplicadas (FLINN, 1921; MILLIKAN, 1926). Tal foco veio acompanhado da formação de engenheiros e técnicos com rigor científico, papel que era atribuído às universidades e às escolas técnicas (GIBBONS; JOHNSTON, 1974). Temos ainda que, temas atuais, como a questão da interdisciplinaridade da pesquisa e a relação entre governo, universidade e indústria na geração e utilização do conhecimento científico, já eram tratados naquele momento (POTTER, 1940), principalmente no período entre guerras (HOLTON, 1962).

\section{AS PRIMEIRAS DÉCADAS DO DEBATE (1960-1989): RELEVÂNCIA DA PESQUISA EM ADMINISTRAÇÃOO}

Nos anos de 1950 se assiste à migração do debate, sobre a relevância social, das Ciências Naturais e Tecnológicas para as Ciências Sociais. A análise dos artigos relacionados às Ciências Naturais e Tecnológicas exterioriza a questão da relevância social como tema presente, porém não central. Por outro lado, nas Ciências Sociais, em geral, e na Administração, em particular, a realidade é outra. De fato, o campo da administração parece ter nascido com uma crise de relevância (HAIRE, 1964).

A inauguração da ciência administrativa como disciplina nos Estados Unidos é marcada pela criação da Operations Reseach Society of America, em 1952, e pelo The Institute of Management Science, em 1953 (HERTZ, 1971; WHITLEY, 1984a). Outro acontecimento importante foi a divulgação dos relatórios de Gordon e Howell (1959) e de Pierson (1959), que criticaram as escolas de administração por não serem úteis para a prática justamente pela falta de rigor científico de suas pesquisas. Esses relatórios deram início a uma virada científica nas escolas de administração, cujos desdobramentos são sentidos até hoje no privilégio dado ao rigor em detrimento da relevância (KEISER; LEINER, 2012). 
A ciência administrativa sempre foi entendida como uma ciência aplicada (THOMPSON, 1956), orientada para a prática (WHITLEY, 1984b), e um campo do conhecimento científico que contribui para o entendimento e a prática da administração (GRUBER; NILES; 1975). No início, a questão premente era a dificuldade de operacionalização dos conceitos oriundos das teorias administrativas (THOMPSON, 1956). Nesse momento, a questão que marcou a discussão sobre a relevância social da pesquisa em administração foi o processo de conversão do conhecimento teórico gerado na universidade em conhecimento prático. Essa dificuldade foi apontada, por alguns autores, como inerente à própria natureza do conhecimento das Ciências Sociais ou ao estágio inicial das teorias administrativas, que dificultava a sua aplicação em situações particulares (GUETZKOW, 1959).

As críticas atuais sobre a falta de relevância social da pesquisa em administração estavam presentes na origem do campo e continuaram presentes nas décadas seguintes. Essas críticas à ciência administrativa, em geral, e às escolas de administração, em particular, nas décadas de 1960, 1970 e 1980, nos Estados Unidos, podem ser atribuídas ao momento histórico, marcado pelos seguintes fatores: primeiro, à necessidade de produção rápida de resultados pelas universidades e pelas escolas de administração, dadas as condições econômicas do país (SMITH; ROBEY; 1973); segundo, o foco da universidade na formação de professores, devido à explosão educacional ocorrida na década de 1950, com menor ênfase na formação de pesquisadores (DRUCKER, 1973); e terceiro, ao desencantamento com os resultados das pesquisas, e com a capacidade de conversão de ciência em aplicação (ALPERT, 1985).

Assim, o sentimento era que, após um quarto de século de desenvolvimento teórico, não havia ocorrido avanço no pensamento gerencial, uma vez que as teorias administrativas não serviam como base para a ação dos gerentes (McGUIRE, 1982). Apontava-se ainda que a ciência administrativa havia atingido uma espécie de platô de desenvolvimento científico e que deveria, então, focar na aplicação, visando solucionar problemas gerenciais (HERTZ, 1971).

Em todo esse período, autores tentaram identificar as causas desse afastamento entre ciência administrativa e a aplicação prática das pesquisas, e propor alternativas para superar a falta de relevância da teoria administrativa para o mundo real. Entre as causas desse estado das coisas, apontavam-se: primeiro, a ignorância, por parte dos pesquisadores, dos problemas reais vividos pelos gestores (MORRIS, 1967; HOWARD, 1968); segundo, a falta de uma ciência administrativa coerente, alinhada com o dia a dia dos gerentes (WHITLEY, 1984a); e terceiro, o cientificismo da teoria administrativa, com ênfase na metodologia e não nas práticas organizacionais. (McGUIRE, 1982) Os gerentes, por outro lado, eram vistos como interessados apenas em resultados simples e rápidos, possuindo uma visão utilitária do conhecimento (BARNES, 1971) e céticos com relação ao conhecimento produzido na academia (DUNCAN, 1974).

\section{A INTENSIFICAÇÃO DO DEBATE (1990-2015): RELEVÂNCIA DA PESQUISA EM ADMINISTRAÇÃOE MODOS DE PRODUÇ̃̃O DE CONHECIMENTO}

O debate sobre a relevância social da pesquisa em administração ganhou novo impulso e novos enfoques a partir dos anos 1990, embora muitos temas presentes no 
debate, desse terceiro período, já tivessem sido tratados em décadas anteriores. O que marca esse período é que a questão da relevância se une à discussão sobre os modos de produção de conhecimento - modo 1 e modo 2, citados anteriormente -, a partir da obra de Gibbons et al. (1994).

Rigor e relevância foram, a partir de então, vistos como pertencentes a lados opostos, embora diversos autores advogassem a possibilidade de produzir conhecimento que fosse igualmente rigoroso e relevante (HODGKINSON; ROUSSEAU, 2009; HODGKINSON; STARKEY, 2011). De fato, para alguns autores rigor e relevância são vistos como objetivos impossíveis de se atingir simultaneamente (KEISER; LEINER, 2009; 2011; RACHE; BEHNAM, 2009). Tais autores baseiam-se na teoria dos sistemas e advogam que há uma suposta incomensurabilidade entre o mundo científico e o mundo da prática, ou seja, cada um destes mundos opera por lógicas ou paradigmas distintos.

Estudos que focam esta questão apontam diferentes razões para tal situação: primeiro, a falta de engajamento dos grupos de interesse na condução e disseminação de pesquisas (STARKEY; MADAN, 2001); segundo, a fragmentação das pesquisas e a distância do contexto de aplicação (TRANFIELD; STARKEY, 1998); e terceiro, dificuldades e problemas que ocorrem nos processos de geração e transferência do conhecimento (VAN DE VEN; JOHNSON, 2006).

O debate sobre os modos de produção de conhecimento, embora tenha recebido bastante destaque, é apenas uma entre várias discussões presentes nesse período que buscam endereçar o dilema do rigor versus relevância da pesquisa em administração (HESSELS; VAN LENTE, 2008). Mas, independentemente da perspectiva, os caminhos apontados para superação desse dilema passam sempre por uma maior aproximação entre universidade e outros agentes, tais como governo e indústria, e por uma forma diferente de relacionamento entre pesquisadores e os usuários das pesquisas.

\section{MAIORES CITADORES}

A análise da rede de citação permite identificar os maiores citadores da rede: são autores que atuam como integradores de conjuntos anteriores de trabalhos. Eles constituem fontes úteis para a compreensão da literatura de um campo científico (GEORGIOU, 2014). 0 interesse pelo debate sobre a relevância da pesquisa em administração cresceu significativamente a partir dos anos 2010, período que concentra o maior número de citadores. Além de oferecerem uma compilação das principais discussões em torno do tema, esses autores realizaram análises críticas que fomentaram o avanço do debate.

O Quadro 2 apresenta os 15 maiores citadores, bem como o número de trabalhos citados por cada um deles. Esse corte foi definido com base na queda expressiva do número de trabalhos citados a partir do $15^{\circ}$. citador. Este critério de corte é usual em estudos de rede de citação (GEORGIOU; HECK, 2015).

Kieser, Nicolai e Seidl (2015), os maiores citadores, realizaram uma revisão sistemática da literatura sobre relevância social da pesquisa em administração, identificando duas correntes principais: a primeira, denominada programática, compreende os estudos que buscam superar a falta de relevância das pesquisas em administração a partir da iden- 
tificação das causas desse problema e da sugestão de soluções; e a segunda, denominada descritiva, que compreende estudos que buscam examinar como a prática da administração se relaciona com os resultados oriundos das pesquisas em administração. Para superar o problema da falta de relevância das pesquisas, os autores apontam para a necessidade de ir além da ideologização que caracteriza o debate atual, bem como evitar a busca de soluções imediatistas que não passam por uma análise crítica e rigorosa sobre como os resultados das pesquisas são utilizados na prática gerencial.

Quadro 2 - Maiores citadores

\begin{tabular}{|l|c|}
\hline Citadores & Número de citados \\
\hline Kieser, Nicolai e Seidl (2015) & 126 \\
\hline Bartunek e Rynes (2014) & 55 \\
\hline Bullinger, Kieser e Schiller-Merkens (2015) & 38 \\
\hline Hodgkinson e Starkey (2011) & 39 \\
\hline Chi Vo, Monoud e Rose (2012) & 34 \\
\hline Nicolai, Schulz e Gobel (2011) & 32 \\
\hline Nicolai e Seidl (2010) & 32 \\
\hline Wolf (2012) & 31 \\
\hline Kieser e Leiner (2012) & 31 \\
\hline Rasche e Behnam (2009) & 30 \\
\hline Hessels e Van Lente (2008) & 29 \\
\hline Van Aken (2005) & 29 \\
\hline Hughes, Bence e Grisoni (2011) & 29 \\
\hline Flickinger, Tuschke e Gruber-Muecke (2014) & 28 \\
\hline Kieser e Leiner (2009) & 27 \\
\hline
\end{tabular}

Bullinger, Kieser e Schiller-Merkens (2015), os terceiros maiores citadores, também realizaram uma revisão sistemática da literatura, concluindo que o dilema entre rigor e relevância tem origem nas diferentes lógicas de pesquisa que buscam, alternativamente, o progresso científico (pesquisa básica) ou o conhecimento relevante (pesquisa aplicada). Ao que, Nicolai, Shulz e Gobel (2011), os sextos maiores citadores, adicionaram que as diferentes visões de mundo entre praticantes e acadêmicos levariam a diferentes interpretações e critérios sobre o que é relevante na prática. (NICOLAI; SHULZ; GOBEL, 2011). Complementarmente, Bartunek e Rynes (2014), os segundos maiores citadores, concluíram em seu trabalho que a discussão ainda é essencialmente centrada na visão de grupos acadêmicos, negligenciando a voz dos praticantes, poucas vezes incorporada nesse debate.

Endereçar essas questões constitui desafio para os estudiosos do campo. Hodgkinson e Starkey (2011), os quartos maiores citadores, realizaram uma análise da produção científica sobre relevância social da pesquisa em administração nas décadas de 1990 e 2000, a partir da qual propuseram uma ênfase em pesquisas Modo 2, tal como advogado também por Tranfield e Starkey (1998) e Starkey e Madan (2001). Contra tal posição, Kieser e Leiner (2012) comentam sobre as diferentes lógicas sob as quais operam acadêmicos e 
praticantes, utilizando tal condição para apontar a inviabilidade de pesquisas colaborativas entre acadêmicos e praticantes e a tentativa de transformar praticantes em copesquisadores.

A partir da análise dos maiores citadores da rede, percebe-se como o debate apresenta algumas posições claramente definidas sobre as razões para o estado atual das coisas. Os problemas que ainda parecem persistir dizem respeito à própria noção do que seja conhecimento relevante (NICOLAI; SEIDL, 2010), e de como visões baseadas em sistemas fechados e autorreferenciados de acadêmicos e praticantes ainda impedem que haja uma maior colaboração entre eles (RACHE; BEHNAM, 2009). Tais sistemas operam segundo lógicas próprias: acadêmicos e praticantes têm definições, explícitas ou implícitas, distintas sobre o que é conhecimento relevante. Nessa perspectiva, acadêmicos só produziriam conhecimento relevante para a prática na medida em que este conhecimento fosse incorporado na própria lógica de uso dos praticantes.

\section{MAIS CITADOS}

A análise da rede de citação também permite identificar os autores mais citados, aqueles que trazem contribuições importantes para o desenvolvimento de um campo científico (GEORGIOU; HECK, 2015). Em geral, eles orientam os principais temas presentes no debate e/ou são atores importantes nas discussões que fazem avançar um campo científico. O Quadro 3 apresenta os 15 autores mais citados, bem como o número de vezes que foram citados na rede. Esse corte foi definido com base na queda expressiva do número de citadores a partir do $15^{\circ}$. citado. Este critério de corte é usual em estudos de rede de citação (GEORGIOU; HECK, 2015).

Entre os 15 artigos mais citados na rede, seis abordam o tema da relevância social da pesquisa em administração sob a ótica dos modos de produção do conhecimento - modo 1 e modo 2 -, o principal eixo do debate a partir dos anos 1990; quatro artigos tratam do papel das escolas de administração no ensino e na prática da administração; três focam as dificuldades para estabelecer uma relação entre a pesquisa e a prática; um artigo foca no uso prático das pesquisas em administração; e um artigo trata do papel dos periódicos científicos e da academia na produção de conhecimento relevante para a sociedade.

É relevante observar que, entre os 15 textos mais citados, seis foram publicados em números especiais de periódicos científicos de alto impacto: quatro no British Journal of Management (BJM); um no Academy of Management Journal (AMJ); e um no Administrative Science Quarterly (ASQ). Os quatro artigos publicados no BJM tratam do texto de Starkey e Madan (2001) que examinou as condições que deram origem às críticas à pesquisa em administração e sua falta de relevância para a prática gerencial. Os autores propõem o Modo 2 de pesquisa como uma solução para superar a falta de relevância social da pesquisa em administração, argumento que se baseia na tese de Gibbons et al. (1994). 
Quadro 3 - Mais citados

\begin{tabular}{|l|c|l|}
\hline Citados & $\begin{array}{c}\text { Número de } \\
\text { citadores }\end{array}$ & Foco Principal \\
\hline Hambrick (1994) & 63 & Papel dos periódicos científicos \\
\hline Starkey e Madan (2001) & 56 & Modo 1/Modo 2 \\
\hline Tranfield e Starkey (1998) & 53 & $\begin{array}{l}\text { Modo 1/Modo 2 e natureza do conheci- } \\
\text { mento em administração }\end{array}$ \\
\hline Rynes, Bartunek e Daft (2001) & 47 & Relação pesquisador-praticante \\
\hline Pfeffer e Fong (2002) & 45 & Escolas de administração \\
\hline Bennis e O'Toole (2005) & 44 & Escolas de administração \\
\hline Ghoshal (2005) & 43 & Escolas de administração \\
\hline Van de Ven e Johnson (2006) & 38 & $\begin{array}{l}\text { Relação entre teoria e prática em } \\
\text { administração }\end{array}$ \\
\hline Huff (2000) & 35 & $\begin{array}{l}\text { Modo 1/Modo 2 e escolas de adminis- } \\
\text { tração }\end{array}$ \\
\hline Beyer e Trice (1982) & 31 & Uso da pesquisa em administração \\
\hline Kieser e Leiner (2009) & 31 & $\begin{array}{l}\text { Relação entre teoria e prática em } \\
\text { administração }\end{array}$ \\
\hline Grey (2001) & 30 & Modo 1/Modo 2 \\
\hline Shapiro, Kirkman e Courtney (2007) & 29 & $\begin{array}{l}\text { Relação entre teoria e prática em } \\
\text { administração }\end{array}$ \\
\hline Pettigrew (2001) & 28 & Modo 1/Modo 2 \\
\hline Hodgkinson, Herriot e Anderson (2001) & Modo 1/Modo 2 \\
\hline
\end{tabular}

Assim como essa tese recebeu muitas críticas, a proposta de Starkey e Madan (2001) também foi alvo de intenso debate. Os autores são acusados de apresentarem uma definição estreita a respeito do debate sobre relevância social e produção do conhecimento, propondo soluções muito particulares (Modo 2), que não resolvem o problema (PETTIGREW, 2001). Além disso, argumenta-se que Starkey e Madan (2001) entendem de maneira equivocada o papel histórico das universidades e das escolas de administração, que deveriam ser independentes e manter uma distância crítica daquilo que é imediatamente relevante para a indústria (GREY, 2001). Complementarmente, o Modo 2 de produção de conhecimento é apontado como uma forma de ciência popularesca, que possui baixo rigor e alta relevância social (HODGINKSON; HERRIOT; ANDERSON, 2001).

Dois dos artigos mais citados têm origem em discursos de abertura no encontro da Academy of Management (HAMBRICK, 1994; HUFF, 2000). O artigo de Hambrick (1994) pode ser lido como uma chamada para a ação de acadêmicos, que deveriam se inserir, se quiserem ser relevantes, no mundo das coisas práticas, acompanhando as mudanças na prática da administração. Huff (2000), por sua vez, discute a relação entre modo 1 e modo 2 de produção de conhecimento, critica essa dicotomia, e propõe o modo 1,5 de produção de conhecimento, que seria uma alternativa às limitações do modo 1 (muito afastado da realidade) e do modo 2 (muito pragmático, afastado da ciência). 
Três entre os artigos mais citados focam a discussão em torno do papel das escolas de administração. Pfeffer e Fong (2002) e Bennis e O'Toole (2005) miram suas críticas na irrelevância das escolas de administração, seja na preparação dos estudantes para o mercado de trabalho, seja na produção de pesquisas que tenham influência sobre a prática gerencial. Ghoshal (2005), por sua vez, foca nas questões éticas envolvidas com a prática da administração e o papel das escolas de administração na preparação de futuros executivos para uma prática gerencial eticamente aceitável.

Esse grupo de autores (maiores citados) conseguiu definir os quatro principais eixos pelos quais o debate sobre rigor versus relevância se desenrolou ao longo dos últimos anos: modos de produção do conhecimento, a relação entre teoria e prática da administração, o papel das escolas de administração, e a relação entre pesquisadores e praticantes. A partir da análise dos problemas de produção e transferência de conhecimento (VAN DE VEN; JOHNSON, 2006), esses autores apontaram caminhos para melhorar o processo de transferência do conhecimento gerado pelas escolas de administração para a prática (SHAPIRO; KIRKMAN; COURTNEY, 2007), ou para evidenciar diferenças entre o mundo da ciência e o mundo da prática administrativa, que impossibilitariam a produção de pesquisas que fossem, ao mesmo tempo, rigorosas e relevantes (KIESER; LEINER, 2009).

\section{ANÁLISE DO CAMINHO PRINCIPAL}

A análise do caminho principal permite identificar a trajetória preferencial em uma rede de citação. É comumente utilizada para verificar como se deu o desenvolvimento de um campo de pesquisa (LIU; LU, 2012). método é detalhado por Georgiou (2014), consistindo em analisar a proporção de caminhos (citações) que passa por um artigo específico, e determinando a sequência de artigos com as maiores proporções. Esta sequência representa, assim, a linha principal de sua literatura constituinte.

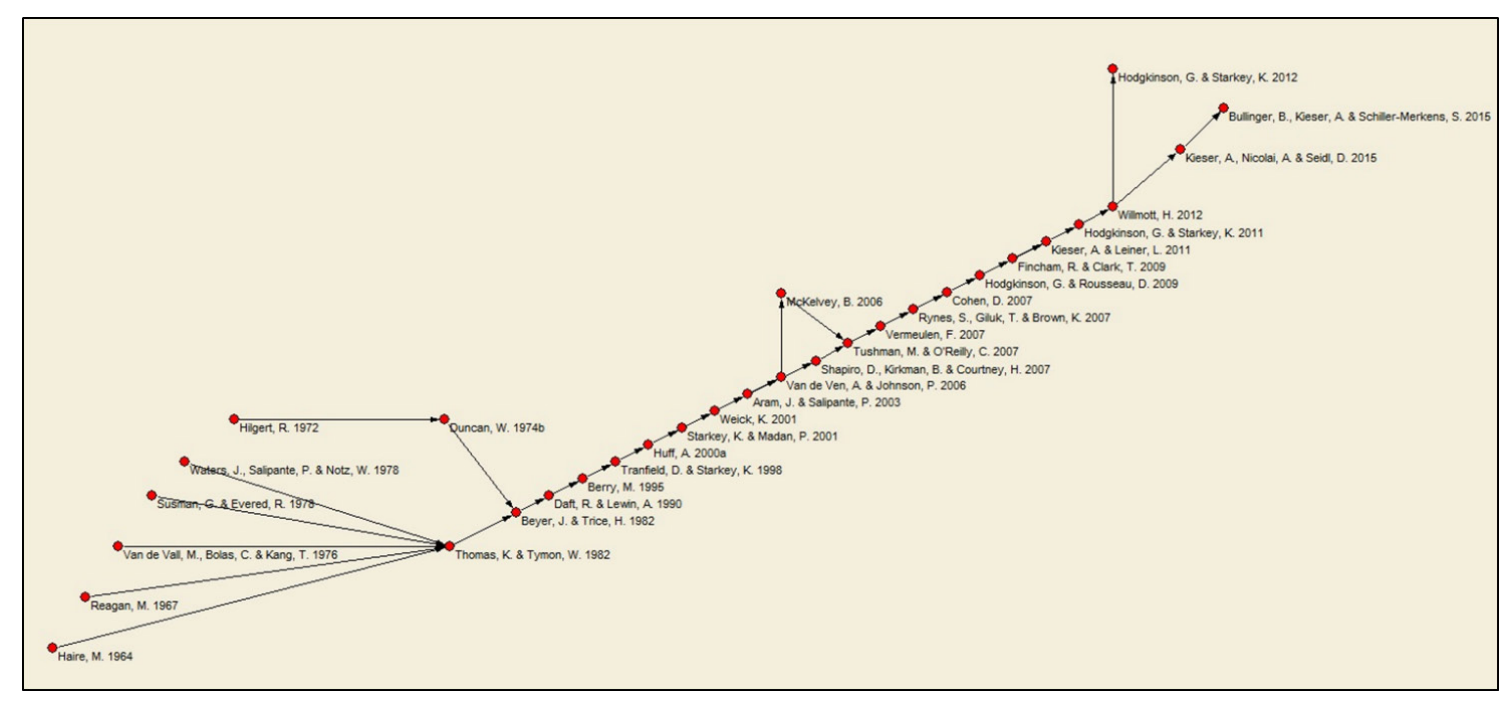

Figura 2 - Análise do caminho principal 
A análise do caminho principal fornece uma visão abrangente da rede de citação, por permitir acompanhar a linha do debate ao longo dos anos. Ela também contribui para fornecer uma lista de leitura inicial, propiciando uma introdução substancial ao restante da rede de citação. Nossa análise resultou em um caminho composto por 31 artigos (4\% da rede), como mostrado na Figura 2. Destacaremos, em seguida, os mais representativos dessa linha de desenvolvimento, sumarizados no Quadro 4.

Quadro 4 - Caminho Principal da Rede de Citação

\begin{tabular}{|c|c|}
\hline Autor & Foco Principal \\
\hline Haire (1964) & Distância entre pesquisa e prática gerencial \\
\hline Reagen (1967) & Pesquisa básica versus pesquisa aplicada \\
\hline Hilgert (1972) & Papel das escolas de administração \\
\hline Duncan (1974) & Distância entre pesquisa e prática gerencial \\
\hline Van de Vall, Bolas e Kang (1976) & Distância entre pesquisa e prática gerencial \\
\hline Susman e Evered (1978) & Pesquisa-ação e intervenção organizacional \\
\hline Waters, Salipante e Notz (1978) & Distância entre pesquisa e prática gerencial \\
\hline Beyer e Trice (1982) & Uso da pesquisa em administração \\
\hline Thomas e Tymon (1982) & Relevância da pesquisa em administração \\
\hline Daft e Lewin (1990) & Relevância da pesquisa em administração \\
\hline Berry (1995) & Relação entre teoria e prática em administração \\
\hline Tranfield e Starkey (1998) & $\begin{array}{l}\text { Modo 1/Modo } 2 \text { e natureza do conhecimento em } \\
\text { administração }\end{array}$ \\
\hline Huff (2000) & Modo 1/Modo 2 e escolas de administração \\
\hline Starkey e Madan (2001) & Modo 1/Modo 2 \\
\hline Weick (2001) & Modo 1/Modo 2 \\
\hline Aram e Salipante (2003) & Relação entre teoria e prática em administração \\
\hline Van de Ven e Johnson (2006) & Relação entre teoria e prática em administração \\
\hline McKelvey (2006) & Relação entre teoria e prática em administração \\
\hline Shapiro, Kirkman e Courtney (2007) & Relação entre teoria e prática em administração \\
\hline Tushman e O'Reilly (2007) & Relevância da pesquisa em administração \\
\hline Vermeulen (2007) & Relevância da pesquisa em administração \\
\hline Rynes, Giluk e Brown (2007) & Relação pesquisador-praticante \\
\hline Cohen (2007) & Relação pesquisador-praticante \\
\hline Hodginkson e Rousseau (2009) & Rigor e Relevância da pesquisa \\
\hline Fincham e Clark (2009) & Rigor e Relevância da pesquisa \\
\hline Kieser e Leiner (2011) & Rigor e Relevância da pesquisa \\
\hline Hodginkson e Starkey (2011) & Modo 1/Modo 2 e rigor vs. relevância da pesquisa \\
\hline Willmott (2012) & Modo 1/Modo 2 e rigor vs. relevância da pesquisa \\
\hline Hodginkson e Starkey (2012) & Rigor e Relevância da pesquisa \\
\hline
\end{tabular}




\begin{tabular}{|l|l|}
\hline Autor & Foco Principal \\
\hline Kieser, Nicolai e Seidl (2015) & Rigor e Relevância da pesquisa \\
\hline Bullinger, Keiser e Schiller-Merkens (2015) & Rigor e Relevância da pesquisa \\
\hline
\end{tabular}

Os artigos da década de 1960 e 1970 apontaram para baixa utilidade do conhecimento produzido pelas ciências administrativas e para a distância entre as escolas de administração e prática gerencial (HAIRE, 1964; HILGERT, 1972). Essas críticas iniciais atribuíam essa situação a problemas como a falta de comunicação entre professores e gerentes, pelo fato daqueles negligenciarem os problemas gerenciais e permanecerem alheios às questões práticas que envolvem o mundo organizacional. Estes, por sua vez, tendiam a ser demasiadamente orientados a resultados, negligenciando contribuições teóricas vindas das escolas de administração. Outros aspectos apontados dizem respeito às diferenças entre o processo de geração de conhecimento administrativo e a sua aplicação em um tempo e local distintos (DUNCAN, 1974), ou a forma como esse conhecimento é gerado, do centro (escolas de administração) para a periferia (gerentes e praticantes). (WATERS; SALIPANTE; NOTZ, 1978).

Entretanto, não se assiste nesse período a uma tentativa de avançar mais profundamente nas origens das questões que se encontram no âmago dessa distância entre o que é ensinado e pesquisado nas escolas de administração e o que se pratica nas organizações. Somente na década de 1980 começa-se a esboçar tentativas para uma definição sobre o que constitui uma pesquisa relevante. Por exemplo, Thomas e Tymon (1982) apontaram cinco componentes da relevância prática da pesquisa, tendo como base as necessidades dos praticantes. Esses estariam relacionados à acurácia dos resultados da pesquisa em capturar o fenômeno vivenciado pelo praticante em seu ambiente organizacional (relevância descritiva), à correspondência entre variáveis investigadas pela teoria e aquilo que o praticante deseja influenciar (relevância de objetivos), à habilidade do praticante de implantar ações a partir da atuação nas variáveis presentes na teoria (validade operacional), à capacidade da teoria de explicar o fenômeno para além do senso comum (não obviedade), e à disponibilidade da teoria para que seja utilizada em tempo útil pelo praticante na solução de seus problemas (oportunidade).

De maneira semelhante, Beyer e Trice (1982) buscaram definir os parâmetros para utilização do conhecimento, levando em consideração as características específicas das organizações. Fundamentalmente, os autores buscaram conectar os comportamentos postos em ação quando se avalia o conhecimento gerado pela pesquisa (avaliação do que é conhecimento relevante, valoração das alternativas de conhecimento, escolha de alternativas, e ação com base na escolha) com os processos organizacionais. Segundo eles, os pesquisadores deveriam tomar conhecimento do modo como as organizações adotam e difundem conhecimento para posicionar suas pesquisas de maneira que essas tenham utilidade.

Ao longo dos anos 1990 e 2000 diversas outras maneiras de definir relevância e de posicionar o debate sobre o impacto social da pesquisa em administração foram apresentadas. Alguns autores criticaram o modelo das Ciências Naturais que é utilizado para conduzir e analisar as pesquisas em Ciências Sociais (DAFT; LEWIN, 1990). Outros 
abordaram a questão dos modos de produção de conhecimento, seja em defesa do Modo 2 (TRANFIELD; STARKEY, 1998; STARKEY; MADAN, 2001), seja criticando essa alternativa (HUFF, 2000; WEICK, 2001).

Ocorreu ainda uma discussão centrada no problema de geração e transferência de conhecimento entre acadêmicos e praticantes (ARAM; SALIPANTE, 2003; VAN DE VEN; JOHNSON, 2006; MCKELVEY, 2006; SHAPRIO; KIRKMAN; COURTNEY, 2007). A colaboração entre acadêmicos e praticantes na produção de conhecimento socialmente relevante passou a ser vista por muitos autores como o principal mecanismo para superar o dilema do rigor versus a relevância (RYNES; GILUK; BROWN, 2007; COHEN, 2007). Entretanto, a falta de consenso entre autores sobre a possibilidade de superação da lacuna entre rigor e relevância parece demonstrar que o debate ainda está não está concluído (FINCHAM; CLARK, 2009; KIESER; LEINER, 2011; HODGKINSON; STARKEY, 2011).

\section{CONCLUSÃO}

O debate sobre a relevância social da pesquisa no campo da administração é longo e multifacetado. Conforme vimos, este debate tem origem na passagem do século XIX para o século XX nas Ciências Naturais e Tecnológicas, tomou grande impulso a partir dos anos 1990 e amadureceu nos anos 2000. Não está concluído. Entretanto, é possível, a partir do exame da literatura gerada, compreender seus contornos e questões centrais. Além disso, observando-se a Figura 2 e o Quadro 4, constata-se tendências temáticas que poderíamos relacionar, de forma geral, à busca da relevância social, ou aplicação prática, da pesquisa. Acreditamos, por isso, que é possível, a partir dos achados da pesquisa, assumir posições e estabelecer cursos de ação. Tal constatação norteará nossa tomada de posição sobre a pesquisa em administração no Brasil, como veremos mais adiante ainda nesta conclusão.

Este estudo buscou contribuir para a criação de um mapa do amplo território de décadas de produção científica. Nós acreditamos que a principal contribuição deste artigo é oferecer uma visão estruturada da literatura, mostrando seu desenvolvimento. Com isso, este estudo oferece àqueles recém-chegados ao debate sobre relevância social da pesquisa em administração um guia para compreensão da vasta literatura existente, fomentando a discussão da questão na comunidade científica brasileira.

A análise da rede de citação revelou que o campo é marcado por polêmicas sobre o que constitui conhecimento relevante, como o conhecimento deve ser gerado, e qual a melhor maneira para transferir conhecimento aos praticantes. Além disso, a análise da rede de citação mostrou as críticas sobre as escolas de administração, antes, dirigidas à baixa produção de conhecimento e à falta de rigor do que era produzido; e, atualmente, por terem sucumbido a uma visão de ciência originada nas Ciências Naturais, relegando a um segundo plano a produção de conhecimento prático.

No Brasil, alguns autores publicaram estudos criticando as fraquezas da produção cientifica local e a orientação produtivista dessa produção (ALCADIPANI, 2011 b; FALASTER et al., 2016). Entretanto, a discussão não parece ter acompanhado o debate internacional. Acreditamos que tal inserção é possível e desejável, pois poderia trazer uma perspectiva 
externa ao debate, que vem se concentrando nos Estados Unidos e na Europa e, ao mesmo tempo, fomentar em nosso país uma visão mais crítica sobre o modo de produção científica.

Considerando os enormes desafios que o país enfrenta, notadamente nos serviços básicos oferecidos ao cidadão, relacionados à educação, saúde, segurança e transporte; e considerando ainda os também enormes desafios que as organizações locais enfrentam para tornar suas operações mais eficazes, eficientes, produtivas, além de socialmente e ambientalmente sustentáveis, pensamos que os pesquisadores locais deveriam direcionar esforços para a pesquisa fundamentados no Modo 2 de geração de conhecimento, ou variações desse modo.

Dessa forma, as escolas locais de administração deveriam: primeiro, fomentar a aproximação com organizações - públicas, privadas e sociais - criando canais permanentes de comunicação e parcerias; segundo, promover a definição de temas relevantes de pesquisa, considerando o estado da arte do conhecimento e as demandas da realidade local; terceiro, buscar a excelência em métodos de geração de conhecimento voltados para a intervenção na realidade, como, por exemplo, a pesquisa-ação (COGHLAN; BRANNICK, 2005; MCNIFF; WHITEHEAD, 2002); quarto, criar ou aperfeiçoar os canais de disseminação do conhecimento, tais como os cursos das próprias escolas, as mídias, a participação em eventos etc.; e, quinto, desenvolver competências relacionadas à advocacy, ou seja, capacitar quadros na identificação de tomadores de decisão e no relacionamento com tais agentes, visando potencializar o potencial de impacto social das pesquisas realizadas.

Defendemos, em suma, uma virada prática para a pesquisa local, sem desconsiderar o valor da pesquisa básica. Acreditamos que essa é a orientação moralmente responsável a ser tomada. Ao mesmo tempo, pensamos que tal orientação aumentaria o potencial de participação da pesquisa brasileira nos fóruns e periódicos internacionais de topo, uma vez que os estudos produzidos teriam forte base empírica e grande relevância social. Complementarmente, poderíamos enriquecer o debate internacional acerca do tema específico da relevância social da pesquisa.

Cabe observar que assumir tal postura não equivale a desconsiderar a questão do rigor científico. Esse, naturalmente, constitui condição necessária, porém não suficiente, para a realização da pesquisa. Alinhamo-nos, portanto, aos autores citados anteriormente que defendem o atendimento do duplo desafio do rigor e da relevância (HUFF, 2000; NICOLAI; SEIDL, 2010).

\section{AGRADECIMENTOS}

Os autores gostariam de agradecer a contribuição substantiva de Joaquim Heck para o desenvolvimento deste artigo, realizando a operação do software Pajek e apoiando as análises dos resultados.

\section{REFERÊNCIAS}

AACSB. The Association to Advance Collegiate Schools of Business. Eligibility Procedures and Accreditation Standards for Business Accreditation. Tampa, Florida: AACSB, 2013. 
AACSB. The Association to Advance Collegiate Schools of Business. Impact of Research: A Guide for Business Schools. Tampa, Florida: AACSB International, 2012.

. The Association to Advance Collegiate Schools of Business. Impact of research task force. Final Report of the AACSB International. Tampa, Florida: AACSB International, 2008.

ALCADIPANI, R. Resistir ao produtivismo: Uma ode à perturbação acadêmica. Cadernos EBAPE.BR (FGV), v. 9, n. 4, p. 1174-1178, 2011 a.

ALCADIPANI, R. A academia e a fábrica de sardinhas. Organizações \& Sociedade, v. 18, n. 57, p. 345-348, 2011b.

ALPERT, D. Performance and paralysis: the organizational context of the American research University. Journal of Higher Education, v. 56, n. 3, p. 241-281, 1985.

ARAM, J.; SALIPANTE, P. Bridging scholarship in management: Epistemological reflections. British Journal of Management, v. 14, p. 189-205, 2003.

BAILEY, B. Can the university aid industry? American Institute of Electrical Engineers Journal, v. 45, p. 742-745, 1926.

BARNES, B. Making out in Industrial Research. Science Studies, v. 1, n. 2, p. 157-175, 1971.

BARTUNEK, J.; RYNES, S. Academics and practitioners are alike and unlike: The paradoxes of academic-practitioner relationships. Journal of Management, v. 40, n. 5, p. 1181-1201, 2014.

BENNIS, W.; O'TOOLE, J. How business schools lost their way. Harvard Business Review, v. 1, p. 96-104, 2005.

BERRY, M. Research and practice of management: A French view. Organization Science, v. 6, p. 104-116, 1995.

BERTERO, C. et al. Produção científica brasileira em administração na década de 2000. RAE-Revista de Administração de Empresas, v. 53, n. 1, p. 12-20, 2013.

BEYER, J.; TRICE, H. The utilization process: A conceptual framework and synthesis for empirical findings. Administrative Science Quarterly, v. 27, p. 591-622, 1982.

BRESNEN, M.; BURRELL, G. Journals à la mode? Twenty years of living alongside Mode 2 and the new production of knowledge. Organization, v. 20, n. 1, p. 25-37, 2012.

BRITISH JOURNAL OF MANAGEMENT. British Academy of Management's 25th Celebration Special Issue, v. 22, n. 3, p. 341-566, 2011.

BULLINGER, B.; KIESER, A.; SCHILLER-MERKENS, S. Coping with institutional complexity: responses of management scholars to competing logics in the field of management studies. Scandinavian Journal of Management, v. 31, n. 3, p. 437-450, 2015.

BUSH, V. Science, the endless frontier: A report to the president. Washington, D.C.: U.S. Government Printing Office, 1945.

CALERO-MEDINA, C.; NOYONS, E. Combining mapping and citation network analysis for a better understanding of the scientific development: The case of the absorptive capacity field. Journal of Infometrics, v. 2, p. 272-279, 2008. 
CARTY, J. The relation of pure science to industrial research. Science, v. 44 p. 511-518, 1916.

CHI VO, L.; MONOUD, E.; ROSE, J. Dealing with the opposition of rigor and relevance from Dewey's pragmatist perspective. Management, v. 15, n. 4, p. 367-390, 2012.

COGHLAN, D.; BRANNICK, T. Doing action research in your own organization. London: Sage, 2005.

COHEN, D. The very separate worlds of academic and practitioner publications in human resource management: Reasons for the divide and concrete solutions for bridging the gap. Academy of Management Journal, v. 50, n. 5, p. 1013-1019, 2007.

COMPTON, K. Specialization and Cooperation in Scientific Research. Science, v. LXVI, n. 1715, p. 435-442, 1927.

DAFT, R.; LEWIN, A. Can organizational studies begin to break out of the normal science straitjacket? An editorial essay. Organization Science, v. 1, n. 1, p. 1-9, 1990.

DRUCKER, P. Science and industry: challenges of antagonistic interdependence. Science, $v$. 204, p. 806-810, 1973.

DUNCAN, W. Transferring management theory to practice. Academy of Management Journal, v. 17, n. 4, p. 724-738, 1974.

ETZKOWITZ, H.; LEYDESDORFF, L. The endless transition: A "triple helix" of university-industry-government relations. Minerva, v. 36, p. 203-208, 1998.

. The triple helix university-industry-government relations: A laboratory for knowledge based economic development. EASST Review, v. 14, p. 14-19, 1995.

FALASTER, C. et al. Motivos de rejeição dos artigos nos periódicos de Administração. Organizações e Sociedade, v. 23, n. 77, p. 285-306, 2016.

FINCHAM, R.; CLARK, T. Can we bridge the rigour-relevance gap? Journal of Management Studies, v. 46, n. 3, p. 510-515, 2009.

FLICKINGER, M.; TUSCHKE, A.; GRUBER-MUECKE, T. In search of rigor, relevance, and legitimacy: what drives the impact of publications? Journal of Business Economics, v. 84, p. 99-128, 2014.

FLINN, A. The Relation of the Technical School to Industrial Research. Science, v. LIV, n. 1404, p. 508-510, 1921.

GARFIELD, E. Is citation analysis a legitimate evaluation tool? Scientometrics, v. 1, n. 4, p. 359-75, 1979.

. Citation indexing for studying science. Nature, v. 227, p. 669-671, 1970.

GEORGIOU, I. Seeing the forest for the trees: An atlas of the politics-administration dichotomy. Public Administration Review, v. 74, n. 2, p. 156-175, 2014.

GEORGIOU, I.; HECK, J. Longitudinal exploratory citation network analysis: an Atlas-based methodology, 2015. Disponível em: http://ssrn.com/abstract=2755389. Acesso em 04 Abr 2016. 
GHOSHAL, S. Bad management theories are destroying good management practices. Academy of Management Learning \& Education, v. 4, p. 75-91, 2005.

GIBBONS, M.; JOHNSTON, R. The roles of science in technological innovation. Research Policy, v. 3, p. 220-242, 1974.

GIBBONS, M. et al. The new production of knowledge: The dynamics of science and research in contemporary societies. London: Sage, 1994.

GORDON, R.; HOWELL, J. Higher education for business. New York, NY: Columbia University Press. 1959.

GREY, C. Re-imagining relevance: A response to Starkey and Madan. British Journal of Management, v. 12, p. S27-S32, 2001.

GUETZKOW, H. Conversion barriers in using social science. Administrative Science Quarterly, v. 4, p. 68-81, 1959.

HAIRE, M. The social sciences and management practices. California Management Review, v. 6, n. 4, p. 3-10, 1964.

HAMBRICK, D. What if the academy actually mattered? Academy of Management Review, v. 19, n. 1, p. 11-16, 1994.

HAUSER, P. Are the social sciences ready? American Sociological Review, v. 11, p. 379384, 1946.

HERTZ, D. Has management science reached a dead end? Innovation, n. 25, p. 44-53, 1971.

HESSELS, L.; VAN LENTE, H. Re-thinking new knowledge production: A literature review and a research agenda. Research Policy, v. 37, p. 740-760, 2008.

HILGERT, R. Business schools fail to communicate with managers: Some proposals for bridging the gap. Business Horizons, v. 15, n. 6, p. 59-63, 1972.

HODGKINSON, G.; HERRIOT, P.; ANDERSON, N. Re-aligning the stakeholders in management research: Lessons from industrial, work and organizational psychology. British journal of Management, v. 12, p. S41-S48, 2001.

HODGKINSON, G.; ROUSSEAU, D. Bridging the rigour-relevance gap in management research: It's already happening! Journal of Management Studies, v. 46, n. 3, p. 534-546, 2009.

HODGKINSON, G.; STARKEY, K. Extending the foundations and reach of design science: further reflections on the role of critical realism. British Journal of Management, v. 23, p. 605-610, 2012.

. Not simply returning to the same answer over and over again: Reframing relevance. British Journal of Management, v. 22, p. 355-369, 2011.

HOLTON, G. Scientific research and scholarship: notes toward the design of proper scales. Daedalus, v. 91, n. 2, p. 362-399, 1962.

HOWARD, R. The practicality gap. Management Science, v. 14, n. 7, p. 503-507, 1968. 
HUFF, A. Changes in organizational knowledge production. Academy of Management Review, v. 25, n. 2, p. 288-293, 2000.

HUGHES, T.; BENCE, D.; GRISONI, L. Scholarship that matters: academic-practitioner engagement in business and management. Academy of Management Learning \& Education, v. 10, n. 1 , p. $40-57,2011$.

HUXLEY, T. Science and Culture and Other Essays. London: Macmillan \& Co, 1888.

JOURNAL OF MANAGEMENT STUDIES. Point-Counterpoint, v. 46, n. 3, p. 510-558, 2009.

KENNELLY, A. The Advancement of Engineering in Relation to the Advancement of Science. Science, v. LXIII, n. 1619, p. 25-29, 1926.

KIESER, A.; LEINER, L. Collaborate with practitioners: But beware of collaborative research. Journal of Management Inquiry, v. 21, n. 1, p. 14-28, 2012.

KIESER, A.; LEINER, L. On the social construction of relevance: A rejoinder. Journal of Management Studies, v. 48, n. 4, p. 891-898, 2011.

KIESER, A.; LEINER, L. Why the rigour-relevance gap in management research is unbridgeable. Journal of Management Studies, v. 46, n. 3, p. 516-533, 2009.

KIESER, A.; NICOLAI, A.; SEIDL, D. The practical relevance of management research: turning the debate on relevance into a rigorous scientific research program. The Academy of Management Annals, v. 9, n. 1, p. 143-233, 2015.

LIMA, G.; WOOD, T. The Social Impact of Research in Business and Public Administration. Revista de Administração de Empresas - RAE, v. 54, n. 4, p. 458-463, 2014.

LIU, J.; LU, L. An integrated approach for main path analysis: Development of the Hirsch index as an example. Journal of the American Society for Information Science and Technology, v. 63, n. 3, p. 528-42, 2012.

MACLAURIN, R. Universities and industries. Journal of Industrial and Engineering Chemistry, v. 8, p. 59-61, 1916.

MARTIN, B. What can bibliometrics tell us about changes in the mode of knowledge production? Prometheus, v. 29, n. 4, p. 455-479, 2011.

MASCARENHAS, A.; ZAMBALDI, F.; MORAES, E. Rigor, relevância e desafios da academia em administração: tensões entre pesquisa e formação profissional. Revista de Administração de Empresas, v. 51, n. 3, p. 265-279, 2011.

MCGUIRE, J. Management theory: Retreat to the academy. Business Horizons, v. 25, n. 4, p. 31-37, 1982.

MCKELVEY, B. Response: Van de Ven and Johnson's "Engaged Scholarship": nice try, but... Academy of Management Review, v. 31, n. 4, p. 822-829, 2006.

MCNIFF, J.; WHITEHEAD, J. Action research: Principles and Practices. New York: Sage, 2002.

MILLIKAN, R. The Practical Value of Pure Science. Science, v. LIX, n. 1514, p. 7-9, 1926.

MORRIS, W. Intuition and relevance. Management Science, v. 14, n. 4, p. 157-165, 1967. 
NEWCOMB, S. Abstract Science in America, 1776-1876. The North American Review, v. 122, n. 250, p. 88-123, 1876.

NICOLAI, A. Bridges to the real "world": applied science fiction or a "schizophrenic tour de force"? Journal of Management Studies, v. 41, n. 6, p. 951-976, 2004.

NICOLAI, A.; SCHULZ, A.; GOBEL, M. Between sweet harmony and a clash of cultures: Does a joint academic-practitioner review reconcile rigor and relevance? The Journal of Applied Behavioral Science, v. 47, n. 1, p. 53-75, 2011.

NICOLAI, A.; SEIDL, D. That's relevant! Different forms of practical relevance in management science. Organization Studies, v. 31, n. 9-10, p. 1257-1285, 2010.

NOOY, W.; MRVAR, A.; BATAGELJ, V. Exploratory social network analysis with Pajek. Cambridge, UK: Cambridge University Press, 2011.

ORGANIZATION STUDIES. Organization Studies as Applied Science: The Generation and Use of Academic Knowledge about Organizations, v. 31, n. 9-10, p. 1189-1207, 2010.

PETTIGREW, A. Management research after modernism. British journal of Management, $v$. 12, p. S61-S70, 2001.

PFEFFER, J.; FONG, C. The end of business schools? Less success than meets the eye. Academy of Management Learning \& Education, v. 1, n. 1, p. 78-95, 2002.

PIERSON, F. The education of American businessmen: A study of University-College programs in business administration. New York, NY: McGraw-Hill, 1959.

POTTER, A. Research and Invention in Engineering College. Science, v. 41, p. 1-7, 1940.

RASCHE, A.; BEHNAM, M. As if it were relevant: A systems theoretical perspective on the relation between science and practice. Journal of Management Inquiry, v. 18, n. 3, p. 243-255, 2009.

REAGAN, M. Basic and applied research: A meaningful distinction? Science, v. 155, p. 1383-1386, 1967.

RYNES, S.; BARTUNEK, J.; DAFT, R. Across the great divide: Knowledge creation and transfer between practitioners and academics. Academy of Management Journal, v. 44, n. 2, p. 340-355, 2001.

RYNES, S.; GILUK, T.; BROWN, K. The very separate world of academic and practitioner periodicals in human resource management: Implications for evidence-based management. Academy of Management Journal, v. 50, n. 5, p. 987-1008, 2007.

SHAPIRO, D.; KIRKMAN, B.; COURTNEY, H. Perceived causes and solutions to the translation problem in management research. Academy of Management Journal, v. 50. n. 2, p. 249-266, 2007.

SMITH, R.; ROBEY, D. Research and applications in operations management: discussion of a paradox. Academy of Management Journal, v. 16, n. 4, p. 647-657, 1973.

STARKEY, K. In defense of modes one, two and three: A response. British Journal of Management, v. 12, p. S77-S80, 2001. 
STARKEY, K.; MADAN, P. Bridging the relevance gap: Aligning stakeholders in the future of management research. British Journal of Management, v. 12, p. S3-S26, 2001.

SUSMAN, G.; EVERED, R. An assessment of the scientific merits of action research. Administrative Science Quarterly, v. 23, n. 4, p. 582-603, 1978.

THOMAS, K.; TYMON, W. Necessary properties of relevant research: Lessons from recent criticisms of the organizational science. Academy of Management Review, v. 7, n. 3, p. 345-352, 1982.

THOMPSON, J. On building an administrative science. Administrative Science Quarterly, v. 1, n. 1, p. 102-111, 1956.

THORPE, R. et al. Rigour, relevance and reward: Introducing the knowledge translation value-chain. British Journal of Management, v. 22, p. 420-431, 2011.

TRANFIELD, D.; STARKEY, K. The nature, social organization and promotion of management research: Towards policy. British Journal of Management, v. 9, p. 341-353, 1998.

TUSHMAN, M.; O'REILLY, C. Research and relevance: implications of Pasteur's quadrant for doctoral programs and faculty development. Academy of Management Journal, v. 50, n. 4, p. 769-774, 2007.

VAN AKEN, J. Management research as a design science: Articulating the research products of mode 2 knowledge production in management. British Journal of Management, v. 16, p. 19-36, 2005.

VAN DE VALL, M.; BOLAS, C.; KANG, T. Applied social research in industrial organizations: an evaluation of functions, theory, and methods. The Journal of Applied Behavioral Science, v. 12 , p. $158-177,1976$.

VAN DE VEN, A.; JOHNSON, P. Knowledge for theory and practice. Academy of Management Review, v. 31, n. 4, p. 802-821, 2006.

VERMEULEN, F. I shall not remain insignificant: Adding a second loop to matter more. Academy of Management Journal, v. 50, n. 4, p. 754-761, 2007.

WATERS, J.; SALIPANTE, P.; NOTZ, W. The experimenting organization: using the results of behavioral science research. Academy of Management Review, v. 3, p. 483-492, 1978.

WEICK, K. Gapping the relevance bridge: Fashions meet fundamentals in management research. British Journal of Management, v. 12, p. S71-S75, 2001.

WHITLEY, R. The fragmented state of management studies: Reasons and consequences. Journal of Management Studies, 21, p. 331-348, 1984a.

. The scientific status of management research as a practically oriented social science. Journal of Management Studies, v. 21, n. 4, p. 369-390, 1984b.

WILLMOTT, H. Reframing relevance as "social usefulness": a comment on Hodgkinson and Starkey's "Not simply returning to the same answer over and over again". British Journal of Management, v. 23, p. 598-604, 2012. 
WOLF, J. How individual scholars can reduce the rigor-relevance gap in management research. German Academic Association for Business Research (VHB), v. 5, n. 2, p. 178-196, 2012.

Data de Submissão: 12/05/2017.

Data de Aprovação: 10/08/2017. 